\title{
Evaluation of the Clinical Features of Infertile Women With Subclinical Hypothyroidism in Relatively High-lodine-Intake Area
}

\author{
Seigo Tachibana ${ }^{\mathrm{a}, \mathrm{b}, \mathrm{g}}$, Kyoko Shirotac ${ }^{\mathrm{c}}$, Tetsuhiro Futata ${ }^{\mathrm{d}}$, Yumi Nagata ${ }^{\mathrm{e}}$, \\ Hiroyuki Yamashita ${ }^{\mathrm{f}}$, Toshihiko Yanase ${ }^{\mathrm{c}}$
}

\begin{abstract}
Background: It is well known that hypothyroidism may cause menstrual abnormalities, infertility, increased risk of miscarriage, obstetric complications, and adverse outcomes in offspring. On the other hand, some studies have reported the efficacy of supplementation of levothyroxine (LT4) for subclinical hypothyroidism $(\mathrm{SCH})$ in infertile women. The aim of this study was to evaluate the clinical features of infertile $\mathrm{SCH}$ patients in Japan.
\end{abstract}

Methods: A total of $156 \mathrm{SCH}$ patients were enrolled in this study. They were divided into two groups: 76 patients who showed positivity for anti-thyroglobulin antibody (TgAb) and/or thyroid peroxidase antibody (TPOAb) were categorized into group A, while 80 patients who showed negativity for both $\mathrm{TgAb}$ and TPOAb were categorized into group B. Between these two groups, we evaluated the following factors: age, thyroid function before treatment, presence of hyperprolactinemia, necessary dose of LT4 for correction of thyroid function, thyroid function after treatment, history of abortion, history of pregnancy after treatment, and dose of LT4 before delivery in pregnant subjects.

Results: All of the above investigated factors showed no significant differences between groups A and B. However, a notable point was that the necessary dose of LT4 for correcting thyroid function did not show a significant difference, regardless of anti-thyroid autoantibody.

Conclusion: In Japan, an area of high iodine intake, in infertile wom-

Manuscript accepted for publication June 04, 2015

${ }^{a}$ Department of Endocrinology, Yamashita Thyroid and Parathyroid Clinic, 1-8 Shimogofukumachi, Hakata-ku, Fukuoka City 812-0034, Japan

${ }^{b}$ Department of Endocrinology and Diabetes Medicine, Fukuoka University Hospital, 7-45-1 Nanakuma, Jonan-ku, Fukuoka City 814-0180, Japan

'Department of Gynecology, Fukuoka University Hospital, 7-45-1 Nanakuma,

Jonan-ku, Fukuoka City 814-0180, Japan

${ }^{\mathrm{d} F u t a t a}$ Tetsuhiro Clinic, Japan

eIVF Nagata Clinic, Japan

fDepartment of Surgery, Yamashita Thyroid and Parathyroid Clinic, 1-8 Shimogofukumachi, Hakata-ku, Fukuoka City 812-0034, Japan

'Corresponding Author: Seigo Tachibana, Department of Endocrinology, Yamashita Thyroid and Parathyroid Clinic, 1-8 Shimogofukumachi, Hakata-

ku, Fukuoka City 812-0034, Japan. Email: Tachibana@kojosen.com

doi: http://dx.doi.org/10.14740/jem285w en with $\mathrm{SCH}$, the administration of an adequate dose of LT4 is recommended, regardless of the presence of chronic thyroiditis.

Keywords: Subclinical hypothyroidism; Infertility; Chronic thyroiditis

\section{Introduction}

It is well known that hypothyroidism may affect the hypothalamic-pituitary-gonadal axis and the peripheral metabolism of sex steroids [1-4]. Hypothyroidism may cause menstrual abnormalities, infertility, increased risk of miscarriage, obstetric complications, and adverse outcomes in offspring [3-5]. Most cases of hypothyroidism are associated with autoimmune thyroiditis, and the prevalence of thyroid autoimmunity among infertile patients was found to be higher than that of a similar age group [6]. In addition, it is known that women with chronic thyroiditis might be at an increased risk of miscarriage and obstetric complications, even in a subclinical hypothyroidism and euthyroid state $[3,7]$.

Some studies have reported the efficacy of supplementation of levothyroxine (LT4) for subclinical hypothyroidism $(\mathrm{SCH})$ in infertile women. In these studies, adequate supplementation of LT4 improved embryo quality and pregnancy outcome $[4,8]$. In Japan, a country with excessive iodine intake, treatment for SCH in infertile women has generally been performed. However, there may be differences in the clinical features of infertile $\mathrm{SCH}$ patients between high iodine intake areas and elsewhere. In this study, we evaluated the clinical features of infertile SCH patients in Japan.

\section{Materials and Methods}

\section{Subjects}

Between January 2009 and December 2012, 417 patients suffering from infertility were introduced to Futata Tetsuhiro Clinic because of a suspicion of thyroid dysfunction detected at screening for the differential diagnosis of infertility. Of these, 156 patients were diagnosed with SCH because the concentra- 
Table 1. The Clinical and Biochemical Profile of Subjects

\begin{tabular}{ll}
\hline Age & $35.26 \pm 4.05$ years \\
TSH & $7.67 \pm 3.38 \mu \mathrm{IU} / \mathrm{mL}$ \\
free T4 & $1.08 \pm 0.18 \mathrm{pg} / \mathrm{dL}$ \\
Anti-thyroid antibody (positive/negative) & $76: 80$ \\
\hline
\end{tabular}

Data are mean \pm SD.

tion of thyroid-stimulating hormone (TSH) was high (TSH > $5.0 \mu \mathrm{IU} / \mathrm{mL}$ ) and free thyroxine (FT4) was in the normal range (reference range: $0.9-1.7 \mathrm{ng} / \mathrm{dL}$ ). These 156 patients were enrolled in this study. The clinical and biochemical profile of the subjects is shown in Table 1.

\section{Treatment}

LT4 was prescribed to these SCH patients. When TSH was corrected to the normal range, the dose of LT4 was continued. In addition, when patients were pregnant, the dose of LT4 was adjusted by TSH concentration as follows. In the first trimester, the target of TSH was below $2.5 \mu \mathrm{IU} / \mathrm{mL}$, and in the second and third trimesters, the target of TSH was below $3.0 \mu \mathrm{IU} / \mathrm{mL}$.

\section{Laboratory measurements}

TSH, FT4, anti-thyroglobulin antibody (TgAb) and thyroid peroxidase antibody (TPOAb) were examined using ECLusys kit (Roche Diagnostics, Penzberg, Germany). The reference ranges were as follows: TSH $0.5-5.0 \mathrm{mIU} / \mathrm{L}$, FT4 $0.9-1.7$ $\mathrm{ng} / \mathrm{dL}, \mathrm{TgAb}<28 \mathrm{IU} / \mathrm{mL}$, and TPOAb $<16 \mathrm{IU} / \mathrm{mL}$.

\section{Study protocol}

This was a retrospective chart review. The total of 156 patients were divided into the following two groups: 76 patients who showed positivity for TgAb and/or TPOAb were categorized into group A, while 80 patients who showed negativity for both $\mathrm{TgAb}$ and TPOAb were categorized into group B. Between these two groups, we evaluated the following factors: age, thyroid function (TSH, FT4) before treatment, presence of hyperprolactinemia (prolactin $>20 \mathrm{ng} / \mathrm{mL}$ ), necessary dose of LT4 for correction of thyroid function, thyroid function (TSH, FT4) after treatment, history of pregnancy after treatment, history of abortion, and dose of LT4 before delivery in pregnant subjects. In addition, these subjects were also divided into the following two groups: 27 patients whose TSH was above $10 \mu \mathrm{IU} / \mathrm{mL}$ were categorized into group $\mathrm{C}$, while 129 patients whose TSH was below $10 \mu \mathrm{IU} / \mathrm{mL}$ were categorized into group $\mathrm{D}$. Between these two groups, we evaluated the following factors: presence of hyperprolactinemia, history of abortion, history of pregnancy after treatment, and dose of LT4 before delivery in pregnant subjects.

\section{Statistical analysis}

In these two groups, statistical analysis was performed using Fisher's exact test and Mann-Whitney's U test. These analyses were performed with JMP ver. 6.0 (SAS Institute Inc.).

\section{Results}

As shown in Table 2, between groups A and B, age, thyroid function (TSH, FT4) before treatment, presence of hyperprolactinemia, and necessary dose of LT4 for correction of thyroid function did not show a significant difference. Thyroid function (TSH, FT4) after treatment, history of abortion, history of pregnancy after treatment, and dose of LT4 before delivery in pregnant subjects also did not show any significant differences. In this retrospective review, all investigated factors showed no significant statistical differences between groups $\mathrm{A}$ and $\mathrm{B}$.

As shown in Table 3, between groups $C$ and $D$, the existence of hyperprolactinemia, history of abortion, and history of pregnancy after treatment also did not show significant differences. Only the dose of LT4 before delivery in pregnant subjects showed a significant difference $(\mathrm{P}=0.01)$.

Table 2. Results of Statistical Analysis Between Groups A and B ( $n=156)$

\begin{tabular}{llll} 
& A & B & P value \\
\hline Age & $35.2 \pm 3.8$ & $35.3 \pm 4.3$ & 1.00 \\
TSH before treatment & $7.91 \pm 4.00$ & $7.44 \pm 2.66$ & 0.59 \\
FT4 before treatment & $1.07 \pm 0.20$ & $1.09 \pm 0.17$ & 0.50 \\
Hyperprolactinemia (+):(-) & $5: 71$ & $6: 74$ & 1.00 \\
Necessary dose of LT4 for correction of thyroid function & $37.83 \pm 21.21$ & $37.50 \pm 21.33$ & 0.85 \\
TSH after treatment & $2.20 \pm 0.93$ & $2.37 \pm 0.92$ & 0.20 \\
FT4 after treatment & $1.29 \pm 0.16$ & $1.31 \pm 0.16$ & 0.55 \\
History of abortion (+):(-) & $24: 52$ & $21: 59$ & 0.48 \\
Pregnancy after treatment (+):(-) & $48: 28$ & $51: 29$ & 1.00 \\
Dose of LT4 before delivery in pregnant subjects & $52.34 \pm 27.94$ & $47.44 \pm 29.13$ & 0.44 \\
\hline
\end{tabular}

Data are mean \pm SD. 
Table 3. Results of Statistical Analysis Between Groups C and D $(n=156)$

\begin{tabular}{llll}
\hline & C & D & P value \\
\hline Hyperprolactinemia (+):(-) & $0: 27$ & $11: 118$ & 0.21 \\
History of abortion $(+):(-)$ & $7: 20$ & $38: 91$ & 0.82 \\
Pregnancy after treatment (+):(-) & $14: 13$ & $86: 44$ & 0.19 \\
Dose of LT4 before delivery in pregnant subjects & $70.31 \pm 23.09$ & $47.02 \pm 28.19$ & 0.01 \\
\hline
\end{tabular}

Data are mean \pm SD.

\section{Discussion}

In this retrospective study, no significant differences between groups A and B were observed. A notable point was that the necessary dose of LT4 for correcting thyroid function did not show a significant difference, regardless of anti-thyroid autoantibody. A consensus about LT4 treatment for infertile women with $\mathrm{SCH}$ and anti-thyroid autoantibody has already been reached; on the other hand, it is controversial whether LT4 treatment for infertile women with $\mathrm{SCH}$ without antithyroid autoantibody should be recommended [9-11]. However, Maruo et al reported that thyroid hormones synergize with FSH to exert direct stimulatory effects on granulosa cell functions, including morphological differentiation, luteinizing hormone/human chorionic gonadotropin (hCG) receptor formation, and steroidogenic enzyme induction, and decreases in ovarian functions during hypothyroidism may account for diminished responsiveness of the granulosa cells to FSH [1]. In addition, Abdel Rahman et al reported that LT4 treatment should be recommended to achieve clinical pregnancies in women with $\mathrm{SCH}$ who are undergoing in vitro fertilizationintracytoplasmic sperm injection [8]. LT4 is one of the safest drugs in that it seldom shows adverse effects, and is particularly cost-effective. Therefore, even if anti-thyroid autoantibodies are negative, appropriate supplementation of LT4 for infertile women with SCH should be considered because the necessary dose of LT4 for correcting thyroid function did not show a significant difference between the anti-thyroid autoantibody-positive and -negative groups.

On the other hand, in several studies, patients with positive $\mathrm{TgAb}$ and/or TPOAb tended to show a high miscarriage rate [12-14]. However, in our study, we could not observe such a tendency. Considering this point, this study was retrospective, and most of our subjects have multiple causes of infertility. In addition, in this series, factors associated with habitual abortion, such as antinuclear antibody and anticardiolipin antibody, were not fully evaluated. These points might have influenced this discrepancy between several reports and our results. Therefore, our retrospective study could not show adequate data associated with anti-thyroid antibody and miscarriage. However, Yoshioka et al reported that, in infertile subjects corrected to a euthyroid state by LT4, there was no difference in the frequency of anti-thyroid autoantibodies between the successfully conceiving group and the unsuccessfully conceiving one. They suggested that the presence of anti-thyroid antibodies might not be a key factor for fertility [15]. Our data are consistent with their report because our subjects were also cor- rected to a euthyroid state by LT4 administration.

In this study, the dose of LT4 before delivery in pregnant subjects did not show a significant difference between subjects with and without chronic thyroiditis. On the other hand, regardless of chronic thyroiditis, group $\mathrm{C}$, which showed a higher titer of TSH before treatment than group D, showed a higher dose of LT4 before delivery than group D. Because this study was retrospective, the causes of SCH besides chronic thyroiditis could not be clarified adequately. However, we suspected that these subjects were probably suffering from mild chronic thyroiditis showing a negative titer of anti-thyroid antibody or polycystic thyroid disease, which is one of the causes of hypothyroidism associated with multiple thyroid cysts influenced by high iodine intake [16]. In addition, high iodine intake might influence their thyroid function because Japan is an area of high iodine intake. Yoshioka et al reported that, in infertile subjects corrected to a euthyroid state by LT4 administration, there was no difference in the frequency of positive anti-thyroid autoantibody between the successfully conceiving group and the unsuccessfully conceiving one. They suggested that the presence of anti-thyroid antibodies might not be a key factor in fertility [15]. Considering their report about the usefulness of LT4 administration for infertile women with $\mathrm{SCH}$ and our results, LT4 should be administered to $\mathrm{SCH}$ patients regardless of the causes of $\mathrm{SCH}$.

In conclusion, in Japan, an area of high iodine intake, in infertile women with $\mathrm{SCH}$, the administration of an adequate dose of LT4 is recommended, regardless of the presence of chronic thyroiditis. In addition, the necessary dose of LT4 did not show remarkable changes associated with the causes of $\mathrm{SCH}$.

\section{Conflicts of Interest}

None of the authors has any potential conflicts of interest associated with this research.

\section{References}

1. Maruo T, Hayashi M, Matsuo H, Yamamoto T, Okada H, Mochizuki M. The role of thyroid hormone as a biological amplifier of the actions of follicle-stimulating hormone in the functional differentiation of cultured porcine granulosa cells. Endocrinology. 1987;121(4):1233-1241.

2. Longcope $\mathrm{C}$, Abend $\mathrm{S}$, Braverman LE, Emerson $\mathrm{CH}$. Androstenedione and estrone dynamics in hypothyroid 
women. J Clin Endocrinol Metab. 1990;70(4):903-907.

3. Poppe K, Glinoer D. Thyroid autoimmunity and hypothyroidism before and during pregnancy. Hum Reprod Update. 2003;9(2):149-161.

4. Kim CH, Ahn JW, Kang SP, Kim SH, Chae HD, Kang BM. Effect of levothyroxine treatment on in vitro fertilization and pregnancy outcome in infertile women with subclinical hypothyroidism undergoing in vitro fertilization/intracytoplasmic sperm injection. Fertil Steril. 2011;95(5):1650-1654.

5. Gerhard I, Becker T, Eggert-Kruse W, Klinga K, Runnebaum $\mathrm{B}$. Thyroid and ovarian function in infertile women. Hum Reprod. 1991;6(3):338-345.

6. Poppe K, Glinoer D, Van Steirteghem A, Tournaye H, Devroey P, Schiettecatte J, Velkeniers B. Thyroid dysfunction and autoimmunity in infertile women. Thyroid. 2002;12(11):997-1001.

7. Kim CH, Chae HD, Kang BM, Chang YS. Influence of antithyroid antibodies in euthyroid women on in vitro fertilization-embryo transfer outcome. Am J Reprod Immunol. 1998;40(1):2-8.

8. Abdel Rahman AH, Aly Abbassy H, Abbassy AA. Improved in vitro fertilization outcomes after treatment of subclinical hypothyroidism in infertile women. Endocr Pract. 2010;16(5):792-797.

9. Abalovich M, Gutierrez S, Alcaraz G, Maccallini G, Garcia A, Levalle O. Overt and subclinical hypothyroidism complicating pregnancy. Thyroid. 2002;12(1):63-68.

10. Abalovich M, Amino N, Barbour LA, Cobin RH, De
Groot LJ, Glinoer D, Mandel SJ, et al. Management of thyroid dysfunction during pregnancy and postpartum: an Endocrine Society Clinical Practice Guideline. J Clin Endocrinol Metab. 2007;92(8 Suppl):S1-47.

11. Garber JR, Cobin RH, Gharib H, Hennessey JV, Klein I, Mechanick JI, Pessah-Pollack R, et al. Clinical practice guidelines for hypothyroidism in adults: cosponsored by the American Association of Clinical Endocrinologists and the American Thyroid Association. Thyroid. 2012;22(12):1200-1235.

12. Iijima T, Tada H, Hidaka Y, Mitsuda N, Murata Y, Amino N. Effects of autoantibodies on the course of pregnancy and fetal growth. Obstet Gynecol. 1997;90(3):364-369.

13. De Vivo A, Mancuso A, Giacobbe A, Moleti M, Maggio Savasta L, De Dominici R, Priolo AM, et al. Thyroid function in women found to have early pregnancy loss. Thyroid. 2010;20(6):633-637.

14. Liu H, Shan Z, Li C, Mao J, Xie X, Wang W, Fan C, et al. Maternal subclinical hypothyroidism, thyroid autoimmunity, and the risk of miscarriage: a prospective cohort study. Thyroid. 2014;24(11):1642-1649.

15. Yoshioka W, Amino N, Ide A, Kang S, Kudo T, Nishihara $\mathrm{E}$, Ito $\mathrm{M}$, et al. Thyroxine treatment may be useful for subclinical hypothyroidism in patients with female infertility. Endocr J. 2015;62(1):87-92.

16. Kubota S, Fujiwara M, Hagiwara H, Tsujimoto N, Takata K, Kudo T, Nishihara E, et al. Multiple thyroid cysts may be a cause of hypothyroidism in patients with relatively high iodine intake. Thyroid. 2010;20(2):205-208. 